\title{
PERCEPÇÃO DE JUSTIÇA ORGANIZACIONAL E INTENÇÃO DE TURNOVER EM EMPRESAS DE AUDITORIA
}

\section{Perception of organizational justice and turnover intention in audit companies}

\author{
Ilse Maria Beuren \\ Dra em Controladoria e Contabilidade pela FEA/USP. Profa do \\ Programa de Pós-Graduação em Contabilidade da Universidade \\ Federal de Santa Catarina (UFSC).email ilse.beuren@gmail.com
}

\section{Flávio Ribeiro}

Doutorando do Programa de Pós-Graduação em Contabilidade na Universidade Federal do Paraná (UFPR). Prof. da Universidade Estadual do Centro-Oeste (UNICENTRO).e-mail:flavioribeiro@unicentro.br

\section{Oscar Lopes da Silva}

Mestre em Contabilidade pela Universidade Federal do Paraná (UFPR). Prof. da Saber e Saber Ltda - A Arte de Educar. email:prof_oscarlopes@saberesaber.com

\section{RESUMO}

O estudo objetiva verificar a relação da percepção de justiça organizacional com satisfação no trabalho, comprometimento organizacional e intenção de turnover de auditores de empresas de auditoria. Uma pesquisa de levantamento foi realizada com os 2.561 auditores cadastrados no Instituto dos Auditores Independentes do Brasil, utilizando-se do questionário traduzido da pesquisa de Parker e Kohlmeyer (2005), e a amostra por acessibilidade compreendeu os 106 respondentes do instrumento de pesquisa. $\mathrm{Na}$ análise dos dados aplicou-se a modelagem de equações estruturais. Os resultados da pesquisa mostram que a percepção de discriminação (justiça organizacional) apresenta relação negativa e significativa com a variável satisfação no trabalho e comprometimento organizacional. Por outro lado, apresenta relação positiva e significativa com a intenção de turnover. Assim, conclui-se que os resultados indicam que a percepção de justiça organizacional dos auditores independentes pesquisados influencia significativamente sua satisfação no trabalho, comprometimento organizacional e intenção de saída da empresa de auditoria.

Palavras-chave: Justiça Organizacional; Satisfação no trabaIho; Comprometimento organizacional; Turnover; Auditores Independentes.

\section{ABSTRACT}

The goal of the study is to verify the relationship of the perception of organizational justice with job satisfaction, organizational commitment and turnover intention of auditors of audit companies. A survey research was conducted with the 2,561 auditors registered at the Brazilian Institute of Independent Auditors, using the questionnaire translated from the research of Parker and Kohlmeyer (2005), and the sample accessibility comprised the 106 respondents from the survey instrument. In the data analysis was applied structural equation modeling. The survey results show that the perception of discrimination (organizational justice) presents a negative and significant relation with the variable of job satisfaction and organizational commitment. On the other hand, shows a positive and significant relation with the intention of turnover. Thus, it is concluded that the results indicate that the perception of organizational justice of surveyed independent auditors significantly influences their job satisfaction, organizational commitment and intention of leaving the audit company.

Keywords: Organizational Justice; Job satisfaction; Organizational commitment; Turnover; Independent Auditors. 


\section{INTRODUÇÃO}

A justiça organizacional foi inicialmente abordada por Homans (1961) e Adans (1965) à luz da justiça distributiva e da teoria da equidade. Esses precursores apresentaram as vertentes sobre as recompensas relativas ao desenvolvimento do trabalho nas organizações. A partir destes estudos pioneiros, vários outros foram realizados ao longo das décadas, particularmente nas áreas da psicologia organizacional e do comportamento organizacional.

A psicologia considera que a justiça é um fenômeno psicossocial e penetra na vida social e organizacional (BEUGRÉ, 1998). O patrimônio mais importante de qualquer organização é sua força de trabalho e o modo como ela é tratada afeta suas atitudes e comportamentos, como o comprometimento, a confiança, o desempenho e a rotatividade. O movimento em direção a uma força de trabalho mais qualificada presume que os trabalhadores mais habilitados passem a reivindicar não apenas melhores empregos, mas também tratamento digno e respeitoso dentro de suas organizações.

As ciências organizacionais consideram que a justiça é construída socialmente e tem influência determinante nas atitudes e comportamentos emitidos no ambiente de trabalho (FOLGER; KONOVSKY, 1989; SKARLICKY; FOLGER, 1997). Percepções como a satisfação no trabalho, com salários, desempenho, intenções de turnover e compromisso organizacional, estão disseminados em diversas pesquisas. Equidade de condições se tornou o foco dos indivíduos. Mesmo com subjetividade na sua interpretação, o que é justo e injusto passou a ser considerado na satisfação ou insatisfação em relação ao trabalho.

Estudos sobre justiça organizacional, como os de Gilliland (1994), Sweeney e McFarlin (1993), subdividem-na e trazem à tona a discussão sobre justiça processual, distributiva e interacional. Essa última, para alguns pesquisadores, como Colquitt $e t$ al. (2001), divide-se em informacional e interpessoal. A interpessoal diz respeito à sensibilidade social, ao grau em que o gestor adota um tratamento - educado, honesto e respeitoso - com as pessoas afetadas pelos procedimentos e decisões distributivas de justiça. A informacional se refere ao fornecimento de informações e explicações acerca das decisões tomadas. Todos esses estudos abordam a percepção de injustiças nas organizações, que provocam falta de comprometimento organizacional, insatisfação com o trabalho e demissões das empresas.

Nos Estados Unidos, Alexander e Ruderman (1987) analisaram a relação entre justiça e resultados organizacionais; Hopkins e Weathington (2006) pesquisaram a percepção de justiça processual e distributiva, confiança, comprometimento, satisfação organizacional e intenção de turnover; Muzumdar (2011) comparou as três dimensões de justiça (processual, distributiva e interacional) com turnover. Em outros países, como no Canadá, Parker e Kohlmeyer (2005) investigaram a relação entre justiça organizacional e intenção de turnover de empresas de contabilidade. Na Malásia, Ponnu e Chuar (2010) estudaram a relação entre justiça organizacional, comprometimento organizacional e intenção de turnover dos empregados. No Paquistão, Ali e Jan (2012) examinaram as dimensões da justiça processual e distributiva e suas relações com comprometimento organizacional e intenção de turnover de companhias médicas e farmacêuticas.

No Brasil, Beuren e Santos (2012) verificaram a percepção de justiça organizacional na avaliação de desempenho dos controllers de empresas que adotam sistemas de remuneração variável. Beuren, Altoé e Dal Vesco (2015) investigaram a relação entre justiça organizacional e comportamento de cidadania organizacional de empregados em uma cooperativa de crédito. Rocha e Paiva (2016) analisaram como se encontram configuradas a percepção de justiça organizacional e as atitudes retaliatórias em relação às dimensões do comprometimento em jovens trabalhadores. Beuren et al. (2016) averiguaram se a percepção de justiça em relação às dimensões dos sistemas de controle gerencial aumenta o comprometimento e a confiança dos gestores. Geremia, Kanan e Marcon (2018) analisaram a relação entre percepção de justiça organizacional e intenção de profissionais de TI permanecerem nas empresas que os empregam.

No entanto, não se identificaram pesquisas que investiguem a percepção de justiça do auditor - profissional que precisa representar as características confiança, qualificação e profissionalismo, esperadas da empresa de auditoria contratada. Esses atributos são reforçados em um cenário de internacionalização 
dos mercados financeiros e de capitais, em consonância com a convergência aos padrões internacionais de contabilidade que o mundo dos negócios está vivenciando, o que deixa a marca do profissional de auditoria como um ator essencial para a credibilidade por parte dos stakeholders.

Embora o turnover crescente de auditores seja considerado um movimento normal, diante de um mercado que sinaliza à competitividade, é importante considerar os atributos esperados dessa mão de obra especializada. A percepção de (in)justiça dos auditores em relação aos processos decisórios que lhes dizem respeito nas empresas de auditoria podem impactar sua confiança, comprometimento, desempenho e rotatividade. Outro fator relevante é que nesse campo ainda não foi apresentado um estudo sobre a razão do turnover - lacuna que motivou a realização desse estudo, que tem o intuito de averiguar a percepção de justiça organizacional dos auditores independentes.

Com base no exposto estabeleceu-se a seguinte pergunta de pesquisa: Qual a relação da percepção de justiça organizacional com a satisfação no trabalho, o comprometimento organizacional e a intenção de turnover de auditores independentes? Assim, o estudo objetiva verificar esta relação. A justiça organizacional, mesmo com estudos já realizados em algumas áreas do conhecimento, ainda é pouco discutida na área da contabilidade no cenário nacional.

Portanto, estudos que analisam o comportamento dos auditores frente às dimensões de justiça organizacional são relevantes em vista de lacunas existentes na literatura. A contribuição do estudo é no sentido de conhecer as possíveis razões do turnover dos profissionais da área de auditoria, ter percepção sobre sua tomada de decisões em relação à permanência ou não nas empresas de auditoria e se tais decisões estão associadas à satisfação no trabalho, ao comprometimento organizacional e/ou à intenção de deixar a empresa.

O estudo justifica-se pela relevância de uma maior compreensão do comportamento das empresas que prestam serviços de auditoria no Brasil, por meio da percepção dos auditores frente às dimensões de justiça organizacional. O auditor independente difere do auditor interno, pela sua independência na realização dos trabalhos e na emissão do parecer de auditoria. Analisar a relação da justiça organizacional e o turnover nessas empresas pode proporcionar este entendimento.

Nesta pesquisa busca-se verificar a percepção de discriminação (justiça organizacional), determinada pela escala desenvolvida por Hunton, Neidermeyer e Weir (1996), e sua relação com (i) a satisfação no trabalho, avaliada com base na escala desenvolvida por Hoppock's (1935) e validada por McNichols, Stahl e Manley (1978), (ii) o comprometimento organizacional, apurado com a utilização da escala construída por Mowday, Steers e Porter (1979), e (iii) a intenção de turnover, mensurada com base na métrica desenvolvida por London e Howat (1978). Estas métricas foram utilizadas por Parker e Kohlmeyer (2005), quando investigaram a relação entre justiça organizacional e intenção de turnover de empregados de empresas de contabilidade, no Canadá.

\section{REFERENCIAL TEÓRICO E HIPÓTESES}

\subsection{Justiça organizacional}

A justiça é um dos fatores inerentes ao bem estar da organização. A preocupação com o que é justo vem sendo alvo de discussões desde tempos remotos. Aristóteles apresentou um modelo de equidade semelhante aos modelos atuais. Platão também abordou o assunto quando escreveu a obra intitulada Politéia (A República). Entretanto, textos datados de épocas mais antigas já apresentavam questionamentos sobre justiça (ALMEIDA; MONTEIRO DA SILVA, 2006).

A justiça, no âmbito organizacional, surgiu em meados da década de 1960, no campo da psicologia social (SOTOMAYOR, 2007), e teve como principais precursores Homans (1961) e Adams (1965). O primeiro lançou os fundamentos do conceito de justiça distributiva, na qual proporcionalidade era sinônimo de justiça. Adams (1965) rompeu a fronteira organizacional com a teoria da equidade, defendendo que a justiça está associada ao direito do indivíduo receber recompensas pelas tarefas realizadas (BEUREN; SANTOS, 2012).

A justiça organizacional tem sido investigada de forma crescente nas últimas décadas, especial- 
mente nas áreas da psicologia organizacional, da gestão de recursos humanos e do comportamento organizacional (CROPANZANO; GREENBERG, 1997). Pesquisadores têm estudado as percepções da justiça organizacional em vários contextos, como a satisfação no trabalho (ALEXANDER; RUDERMAN, 1987; PARKER; KOHLMEYER, 2005; CHOI, 2011), a satisfação com o salário (FOLGER; KONOVSKY, 1989), o desempenho (COHEN-CHARASH, 2001; BEUREN; SANTOS, 2012), a intenção de turnover (PARKER; KOHLMEYER, 2005; GEREMIA; KANAN; MARCON, 2018), o compromisso organizacional (ALEXANDER; RUDERMAN, 1987; PARKER; KOHLMEYER, 2005; CHOI, 2011; JESUS; ROWE, 2015); as atitudes retaliadoras (ROCHA; PAIVA, 2015); a confiança dos gestores (BEUREN et al., 2016).

De forma abrangente, a justiça organizacional envolve a percepção dos membros da organização quanto à equidade de suas condições (FOLGER; CROPANZANO, 1998). A justiça organizacional é atribuída à percepção do indivíduo sobre o que é justo ou injusto na organização (OMAR, 2006). Entretanto, conforme Beuren e Santos (2012), a percepção do indivíduo em relação à justiça é cercada de subjetividade, uma vez que o que é justo para um indivíduo pode não ser para outro. A justiça organizacional está atrelada à clareza com a qual os indivíduos são avaliados. Segundo Locke (1976), o viés no processo decisório pode provocar insatisfação no trabalho e falta de comprometimento organizacional.

Alguns teóricos (ALEXANDER; RUDERMAN, 1987; PARKER; KOHLMEYER, 2005) apresentam dois tipos de justiça organizacional: justiça processual e distributiva. Entretanto, essa divisão não é unânime na academia. Outros autores (GIRAUD; LANGEVIN; MENDONZA, 2008) defendem a existência de três dimensões de justiça: processual, distributiva e interacional. Ainda há outros (REGO, 2000; ASSMAR; FERREIRA; SOUTO, 2005; SOTOMAYOR, 2006; 2007) que reconhecem quatro dimensões, subdividindo a justiça interacional em informacional e interpessoal.

A primeira dimensão de justiça organizacional abordada na literatura foi a justiça distributiva, definida como a equidade conferida nos resultados (SOTOMAYOR, 2006). Cohen-Charash e Spector (2001) afirmam que a justiça distributiva trata da justiça baseada em resultado e tem o potencial de implicar fortemente no contexto organizacional, em decorrência da importância da distribuição de resultados.

A origem da justiça distributiva remonta à teoria de troca no comportamento social de Homans (1961). Essa teoria explica a relação de troca entre os indivíduos em duas perspectivas: as recompensas proporcionais aos custos incorridos e os lucros proporcionais aos investimentos (SOTOMAYOR, 2006). Entretanto essa teoria possuía falhas, principalmente em relação à subjetividade na interpretação de cada indivíduo sobre recompensa, custo, lucro e investimento.

Fundamentado nessas ideias, Adams (1965) elaborou a teoria da equidade no contexto organizacional. Para avaliar a equidade, avaliava o valor dos insumos do trabalho dos indivíduos em relação aos resultados recebidos pela organização (PARKER; KOHLMEYER, 2005). Entretanto, a principal preocupação de Adams era perceber quando e porque as relações de troca entre empregados e empregadores eram compreendidas como justas ou injustas pelos empregados e quais seriam suas reações. Por isso, apesar de ser conhecida como a teoria da equidade, a iniquidade era o principal foco do estudo.

A teoria da equidade e demais modelos da justiça distributiva não alcançaram a explicação completa das reações pessoais frente à percepção da injustiça. Atribuía o principal motivo das reações negativas nas organizações aos fatores relacionados com a distribuição e alocação de recompensas, utilizando um modelo simplista, incapaz de considerar fatores sociais inerentes à percepção justa ou injusta das recompensas (ALMEIDA; MONTEIRO DA SILVA, 2006). Assim, em meados da década de 70, pesquisadores perceberam que a justiça no resultado não era a única fonte de interesse dos indivíduos, pois estavam interessados na justiça do processo que gerava esses resultados (SOTOMAYOR, 2007).

Emergiu, assim, uma nova dimensão de justiça organizacional, a justiça processual, definida por Greenberg (1990) como a justiça decorrente das políticas e dos procedimentos utilizados na tomada de decisões. Precursores dos estudos da justiça processual, como Thibaut e Walker (1975), buscaram interpretar as reações dos indivíduos frente a diferentes formas de procedimentos legais. Entretanto, foi apenas com 
Leventhal (1980) que a justiça processual foi conduzida para o contexto organizacional. Leventhal (1980) apontou seis critérios a serem adotados para que um procedimento seja considerado justo: consistência, não enviesamento, exatidão das informações, correção, representatividade e eticidade.

Segundo Parker e Kohlmeyer (2005), a consistência é um dos principais critérios para avaliar a equidade dos procedimentos de alocação em grupos sociais. Com base nesse critério, os procedimentos de alocação utilizados para determinar as recompensas devem ser consistentes entre os indivíduos ao longo do tempo. Desse modo, é necessária a aplicação de procedimentos semelhantes a todos os indivíduos sem conceder vantagem especial a ninguém.

Tyler e Lind (1988) sugerem o modelo de valor do grupo como tentativa para explicar a importância da justiça processual para os indivíduos dentro da organização, a premissa é a natureza social dos seres humanos. Isso revela que a participação em grupos sociais promove importantes recompensas psicológicas: indivíduos buscam ser valorizados e aceitos pelo grupo e, quando isso ocorre, há um aumento da autoestima.

Conceber a organização como um grupo altamente social implica considerar que os empregados são sensíveis ao nível de respeito que recebem. Assim, de acordo com o modelo de Tyler e Lind (1988), o respeito e o valor da organização para com o funcionário são refletidos pela equidade processual atribuída na tomada de decisões, uma vez que decisões justas revelam a valorização do indivíduo e a proteção dos seus direitos na organização. Para Parker e Kohlmeyer (2005), a percepção da injustiça denota que o funcionário não é digno de respeito pela empresa.

Paralelamente à dimensão de justiça processual, Bies e Moag (1986) introduziram uma terceira vertente no modelo de justiça organizacional, a justiça interacional, que se refere à percepção das pessoas em relação à qualidade do tratamento recebido nos procedimentos organizacionais (MAIA, 2005). A justiça interacional reflete a qualidade da interação com os gestores (REGO; SOUTO, 2004), incluindo aspectos como tratamento digno aos empregados e explicações sobre as decisões tomadas.

Greenberg (1993) e Cropanzano e Greenberg (1997) sugerem a distinção da vertente interacional em duas facetas: interpessoal e informacional. A justiça interpessoal representa o grau em que as pessoas são tratadas com educação, dignidade e respeito pelas autoridades envolvidas no processo ou a determinação das recompensas (COLQUITT et al., 2001). Por sua vez, a justiça informacional reflete o fornecimento de informações e a explicação sobre as decisões tomadas em âmbito organizacional (REGO; SOUTO, 2004).

Embora a justiça, em suas distintas dimensões, seja essencial para a existência de um ambiente harmônico e produtivo nas organizações, estudos como os de Mikula (1986) indicam que locais de trabalho são ambientes com o maior número de casos de injustiça. Injustiças tendem a se refletir no desempenho, na satisfação e no comprometimento dos empregados em relação à empresa (ALMEIDA; MONTEIRO DA SILVA, 2006). Empregados que percebem um ambiente justo tendem a responder com atitudes positivas, comprometimento com a organização, satisfação com o trabalho e baixa intenção de saída da empresa (PARKER; KOHLMEYER, 2005).

\subsection{Justiça organizacional e intenção de turnover}

O Turnover é caracterizado pelo movimento de admissão e desligamento de profissionais na organização. Também denominado de rotatividade de pessoal, o turnover representa a intenção dos indivíduos em deixarem a empresa em prol de melhores condições de trabalho (BORGES; RAMOS, 2011). Em várias organizações o turnover é visto como um problema, uma vez que gera custos ao empregador, principalmente quando a organização investe intensivamente no treinamento de seus empregados (CAMPOS; MALIK, 2008).

Konovsky e Cropanzano (1991) descrevem que a percepção da justiça organizacional pelos funcionários tende a reduzir a tendência de turnover nas organizações, ou seja, empregados que percebem que a organização é justa na tomada de decisões que lhe dizem respeito não tendem a demonstrar interesse em sair da empresa.

A relação entre a percepção de justiça organizacional e a intenção de saída (turnover) de indivíduos de empresas é identificada em artigos publicados 
na literatura internacional, mas não se encontraram estudos com este foco em âmbito nacional. Os estudos encontrados por meio da busca pelas palavras "Organizational Justice", "Distributive Justice", "Pro- cedural Justice" e "Turnover", nas bases de dados do Portal de Periódicos da CAPES e do Portal Scientific Electronic Library Online (SciELO), são evidenciados no Quadro 1.

Quadro 1 Estudos empíricos sobre a percepção de justiça organizacional e o turnover de indivíduos

\begin{tabular}{|c|c|c|}
\hline Autores & Periódicos & Temática abordada e resultados das pesquisas \\
\hline $\begin{array}{l}\text { Alexander e } \\
\text { Ruderman (1987) }\end{array}$ & Social Justice Research & $\begin{array}{l}\text { Analisaram a relação entre justiça e os resultados organizacionais em seis instituições } \\
\text { federais dos Estados Unidos, tendo como amostra cerca de } 2.800 \text { pessoas. Os resultados } \\
\text { demonstraram haver relação significativa entre as dimensões de justiça processual e distri- } \\
\text { butiva e a satisfação com o trabalho, confiança na gestão e intenção de saída da empresa. }\end{array}$ \\
\hline $\begin{array}{l}\text { Parker e } \\
\text { Kohlmeyer (2005) }\end{array}$ & $\begin{array}{l}\text { Accounting, } \\
\text { Organization } \\
\text { and Society }\end{array}$ & $\begin{array}{l}\text { Investigaram a relação entre justiça organizacional e intenção de saída de profissionais de } \\
\text { empresas de contabilidade. Os resultados, em uma amostra de } 76 \text { contadores cana- } \\
\text { denses, sugerem que a percepção de justiça organizacional influencia na intenção dos } \\
\text { indivíduos saírem da empresa na qual trabalham, apresentando uma relação inversa com } \\
\text { o nível de comprometimento organizacional e satisfação com o trabalho. }\end{array}$ \\
\hline $\begin{array}{l}\text { Hopkins e } \\
\text { Weathington (2006) }\end{array}$ & $\begin{array}{l}\text { The Journal of } \\
\text { Psychology }\end{array}$ & $\begin{array}{l}\text { Pesquisaram a percepção de justiça processual e distributiva, confiança, comprometi- } \\
\text { mento, satisfação organizacional e intenção de saída da organização. O estudo foi realiza- } \\
\text { do em uma empresa de transporte dos EUA com aproximadamente } 34.500 \text { empregados } \\
\text { e faturamento anual de aproximadamente } 8 \text { bilhões de dólares. A amostra foi constituída } \\
\text { por } 500 \text { empregados selecionados aleatoriamente. Os resultados sugerem que a relação } \\
\text { entre justiça distributiva e satisfação organizacional é mediada em parte pela confiança } \\
\text { dos empregados nos seus gestores. Essa característica também foi observada na relação } \\
\text { entre a justiça processual e pretensão dos empregados de sair da empresa. }\end{array}$ \\
\hline Ponnu e Chuar (2010) & $\begin{array}{l}\text { African Journal of } \\
\text { Business Management }\end{array}$ & $\begin{array}{l}\text { Averiguaram a relação entre justiça organizacional, comprometimento organizacional e } \\
\text { intenção de saída dos empregados em empresas da Malásia. Com uma amostra de } 172 \\
\text { individuos, os resultados demonstram que tanto a percepção de justiça distributiva como } \\
\text { processual contribuem de forma significativa para explicar o comprometimento organiza- } \\
\text { cional e a intenção dos funcionários em deixarem a empresa. }\end{array}$ \\
\hline Muzumdar (2011) & $\begin{array}{l}\text { Journal of Behavioral } \\
\text { Studies in Business }\end{array}$ & $\begin{array}{l}\text { Relacionaram as três dimensões de justiça (processual, distributiva e interacional) com o } \\
\text { comportamento dos funcionários de sair da empresa. Foi aplicado um questionário com- } \\
\text { posto por sete questões para } 280 \text { empregados de duas empresas distintas dos EUA. Os } \\
\text { resultados revelaram uma alta relação entre a média das questões de justiça interacional } \\
\text { e o turnover dos empregados nas duas empresas. }\end{array}$ \\
\hline Ali e Jan (2012) & $\begin{array}{l}\text { Journal of } \\
\text { Managerials } \\
\text { ciences }\end{array}$ & $\begin{array}{l}\text { Examinaram as dimensões da justiça processual e distributiva e suas relações com o } \\
\text { comprometimento organizacional e a intenção de saída de companhias médicas e farma- } \\
\text { cêuticas do Paquistão. Em uma amostra de } 223 \text { funcionários, os resultados indicaram uma } \\
\text { relação significativa entre a percepção de justiça processual e distributiva e o comprome- } \\
\text { timento organizacional e intenção de saída da empresa. }\end{array}$ \\
\hline
\end{tabular}

Fonte: Elaboração própria.

No Quadro 1, observam-se diversos estudos empíricos sobre a percepção de justiça organizacional e a intenção dos indivíduos de deixar a empresa, realizados em diferentes países. As pesquisas, de modo geral, demonstraram haver relação significativa entre as diferentes dimensões de justiça e o turnover. Entretanto, entre os resultados dessas pesquisas, não há conformidade sobre quais dimensões demonstraram uma relação mais intensa com a variável turnover. O estudo de Parker e Kohlmeyer (2005) será mais detalhado, uma vez que inspira e fundamenta vários elementos da presente pesquisa.

Parker e Kohlmeyer (2005) analisaram a relação entre a percepção de justiça organizacional e a intenção de saída (turnover) dos indivíduos da organização. A amostra foi constituída por três das cinco maiores empresas de contabilidade do Canadá. Ao todo, foram distribuídos 135 questionários para os funcionários das empresas, dos quais 81 retornaram. Desses, cinco indivíduos não responderam todo o questionário, sendo os mesmos retirados do estudo, 
totalizando 76 questionários válidos, um percentual de $56 \%$ de respondentes. Com o propósito de evitar tendências de respostas, foram distribuídas duas versões de questionário com a ordem das questões alteradas, mas não houve diferença significativa entre as versões para qualquer variável.

As variáveis utilizadas no estudo compreenderam: intenção de turnover, percepção de discriminação, comprometimento organizacional e satisfação com o trabalho. Para mensurá-las, o instrumento de pesquisa foi subdividido em quatro blocos, cada um representado por um conjunto de assertivas. A partir do construto teórico, estabeleceram-se e testaram-se três hipóteses de pesquisa. Para confirmar ou rejeitar as hipóteses, foi inicialmente utilizada a análise de regressão linear. Posteriormente, como forma de análise complementar, foi empregada a Modelagem de Equações Estruturais (Structural Equation Modeling - SEM). Os resultados da pesquisa indicaram que a percepção de justiça influencia a satisfação no trabalho, o comprometimento organizacional e a intenção de saída do funcionário da empresa.

Ressalta-se que nesta pesquisa adotaram-se as hipóteses, as variáveis e o instrumento de pesquisa do estudo de Parker e Kohlmeyer (2005). Esse procedimento foi adotado em razão da similaridade entre os objetivos de ambos os estudos. Entretanto, destaca-se que a presente pesquisa difere do estudo de Parker e Kohlmeyer (2005) na amostra, uma vez que se investiga a percepção de justiça de auditores independentes, além de diferir do contexto organizacional, uma vez que neste estudo a pesquisa foi aplicada no Brasil.

\subsection{Desenvolvimento das hipóteses}

De acordo com Coher-Charash e Spector (2001), percepção de justiça organizacional repercute positivamente nos funcionários, aumentando a sua satisfação no trabalho e, consequentemente, os incentivando a estarem mais comprometidos com os interesses da organização. Para Locke (1976), a remuneração e as oportunidades de promoção são fundamentais para a satisfação no trabalho. Entretanto, o viés no processo de tomada de decisões pode implicar em desrespeito organizacional e insatisfação dos empregados. Isso leva à seguinte hipótese:
$\mathrm{H}_{1}$ : A percepção de discriminação na tomada de decisões é negativamente associada com a satisfação no trabalho.

Para Angle e Perry (1981), o comprometimento organizacional, que se trata de quando o empregado está disposto a despender considerável esforço em nome da organização, é caracterizado pela forte aceitação das crenças e dos objetivos da empresa. O comprometimento organizacional tem sido considerado um fator essencial na compreensão e na explicação do comportamento associado ao trabalho dos empregados nas organizações (BAKHSHI; KUMAR; RANI, 2009).

Entretanto, uma vez que decisões tendenciosas são percebidas pelos empregados, há diminuição no comprometimento organizacional (PARKER: KOHLMEYER, 2005). A seguinte hipótese resume esses argumentos:

$\mathrm{H}_{2}$ : A percepção de discriminação na tomada de decisões é negativamente associada com o comprometimento organizacional.

Conceber que a justiça é um fator essencial para o bem estar organizacional significa considerar que a percepção da injustiça na tomada de decisões pode converter-se em atitudes negativas dos empregados e aumento da rotatividade na empresa (COHER-CHARASH; SPECTOR, 2001). Os indivíduos, quando percebem a existência de decisões tendenciosas, podem ser motivados a buscar emprego em outras empresas, que supostamente os respeitem e proporcionem recompensas justas. Assim, formulou-se a seguinte hipótese:

$\mathrm{H}_{3}$ : A percepção de discriminação na tomada de decisões é positivamente associada com a intenção do indivíduo deixar a empresa.

\section{MÉTODO E PROCEDIMENTOS DA PESQUISA}

Este estudo, caracterizado como descritivo, foi realizado por meio de pesquisa de levantamento ou survey e com abordagem quantitativa do problema. Nesse contexto, o estudo descreve a relação da justiça organizacional e do turnover de auditores que trabalham em empresas de auditoria no Brasil. 


\subsection{Instrumento de pesquisa}

Para realizar a coleta de dados utilizou-se o questionário da pesquisa de Parker e Kohlmeyer (2005), que foi traduzido e submetido a adaptações de nomenclatura devido às diferenças na amostra de ambas as pesquisas. $\mathrm{O}$ instrumento de pesquisa compõe-se de quatro blocos, sendo cada um conduzido para a determinação de uma variável (Apêndice A).

As questões foram traduzidas da pesquisa de Parker e Kohlmeyer (2005) e utilizadas neste estudo. A variável intenção de turnover foi mensurada pela métrica criada e validada por London e Howat (1978). Para avaliar a percepção de discriminação dos indivíduos utilizaram a métrica de três itens, desenvolvida por Hunton, Neidermeyer e Weir (1996). O comprometimento organizacional foi mensurado com base na métrica de nove itens, desenvolvida e validada por Wowday, Steers e Porter (1979). A satisfação no trabalho foi determinada com base na métrica de quatro itens, elaborada por Hoppock (1935) e que, segundo Parker e Kohlmeyer (2005), é uma das mais utilizadas na literatura. A escala de respostas dos três primeiros blocos é de sete pontos do tipo Likert, que vai de (1) discordo totalmente até (7) concordo totalmente.

A adaptação no instrumento de pesquisa do estudo de Parker e Kohlmeyer (2005) se restringe, além de adaptações de nomenclatura em função da mudança de campo, a ajustes de redação recomendados nos pré-testes. O primeiro teste foi realizado com um aluno de Mestrado em Ciências Contábeis, a fim de detectar problemas de ambiguidade e coerência nos termos adotados. O segundo teste foi aplicado a um professor que ministra a disciplina de auditoria, com vistas a identificar possíveis dificuldades na compreensão das questões. O terceiro teste foi realizado com um profissional da área de auditoria, para verificar as dificuldades do respondente frente ao questionário, sendo o mesmo excluído da amostra final.

\subsection{População e amostra da pesquisa}

Parker e Kohlmeyer (2005) investigaram empresas de contabilidade canadenses. A seleção dessa população deu-se por evidências empíricas de que funcionários de empresas de contabilidade tendem a reagir negativamente frente à percepção de injustiça organizacional. Neste sentido, porém com uma população diferente, o presente estudo analisa a percepção de justiça organizacional de auditores independentes.

A opção por investigar esta categoria de profissionais, os auditores independentes, decorre da importância do papel do auditor no mercado de capitais e do desafio das empresas de auditoria na busca de mão de obra qualificada. Os auditores podem deixar a empresa em que trabalham se perceberem que ela se utiliza de métodos e procedimentos injustos nas decisões de avaliação de desempenho.

O universo deste estudo compreende os 2.561 auditores cadastrados no Instituto dos Auditores Independentes do Brasil (IBRACON) e que atuam em empresas de auditoria. Para esses auditores foi enviado o questionário em setembro de 2013, cujo procedimento foi repetido mensalmente até dezembro de 2013. A amostra corresponde aos 106 respondentes do instrumento de pesquisa, desse modo se caracteriza como uma amostra por acessibilidade.

A determinação da amostra é um ponto de discussão na aplicação da modelagem de equações estruturais. Loehlin (2004) indica que a amostra deve apresentar entre 100 e 200 observações, enquanto que Bentler e Chou (1987) descrevem que há necessidade de no mínimo cinco observações por variável analisada. Stevens (2002) destaca que a presença de 15 observações por preditor permite validade estatística para a análise. Pode-se ainda aplicar alguns métodos específicos na determinação da amostra, como o uso da tabela Cohen (HAIR et al., 2014) e o emprego do software $G^{\star}$ Power (RINGLE; SILVA; BIDO, 2014). Nesta pesquisa adotaram-se os critérios indicados por Bentler e Chou (1987), Stevens (2002) e Loghlin (2004) para sustentar a determinação da amostra com 106 observações.

\subsection{Procedimentos de análise dos dados}

Para proceder à análise dos dados, replicou-se neste estudo o tratamento conferido às variáveis na pesquisa de Parker e Kohlmeyer (2005). Na Figura 1, demonstra-se a disposição das variáveis em relação a representação gráfica das hipóteses da pesquisa.

Observa-se na Figura 1 a presença da variável percepção de discriminação como variável dependente do modelo. As hipóteses da pesquisa (H1, 
H2, H3) destacam a relação entre a percepção de discriminação e as variáveis satisfação no trabalho, comprometimento organizacional e intenção de saída. A satisfação no trabalho e comprometimento organizacional podem impactar na intenção de saída do indivíduo da empresa.

Para proceder à análise dos dados, seguindo a metodologia proposta por Parker e Kohlmeyer (2005), utilizou-se o modelo de regressão linear e a modelagem de equações estruturais. O modelo de regressão linear foi aplicado com a finalidade de identificar a relação entre a percepção de discriminação e as demais variáveis do estudo.

A modelagem de equações estruturais foi adotada como forma de identificar a relação causal entre as variáveis. Conforme Brei e Liberali Neto (2006), a modelagem de equações estruturais tem como principal função especificar e estimar os modelos de regressões lineares entre variáveis. Neste sentido, buscou-se verificar se as variáveis satisfação no trabalho e comprometimento organizacional influenciam a intenção de turnover dos indivíduos.

Figura 1 Representação gráfica das hipóteses da pesquisa

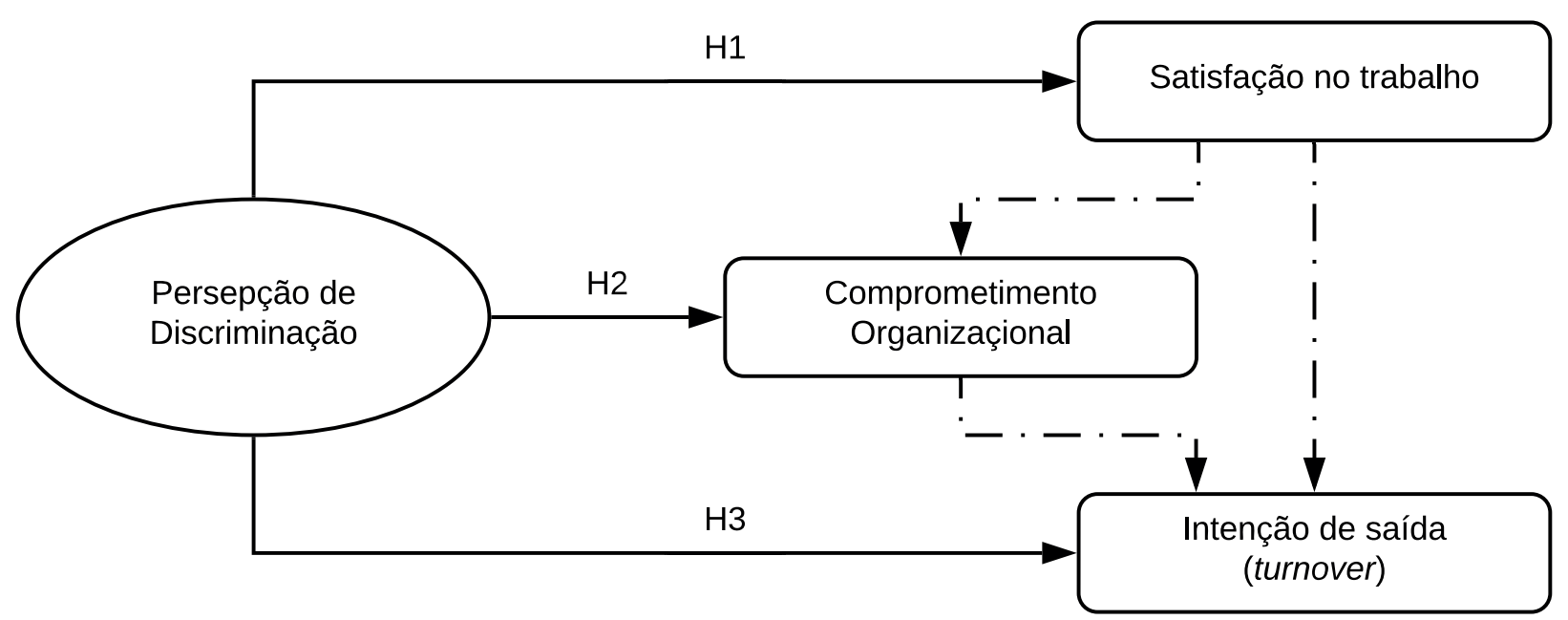

Fonte: Adaptado de Parker e Kohlmeyer (2005).

\section{DESCRIÇÃO E ANÁLISE DOS REŞULTADOS}

\subsection{Dados demográficos}

Na Tabela 1 apresentam-se os dados demográficos dos auditores independentes respondentes do instrumento de pesquisa.

A priori, os resultados da Tabela 1 indicam que na área de auditoria, considerando a amostra pesquisada, há predominância de auditores do gênero masculino (82\%) e concentração entre as faixas de idade de 41 a 45 anos $(21,70 \%)$ e 25 anos ou menos $(20,75 \%)$. Aproximadamente $75 \%$ da amostra tem formação acadêmica na área de Ciências Contábeis, e cerca de $47 \%$ possuem nível de escolaridade superior a graduação, sugerindo alta qualificação. Nota-se ainda que a maior parcela dos auditores (56\%) atua na empresa na faixa de 5 anos ou menos, apontando alta rotatividade.

\subsection{Análise de Modelagem de Equações Estruturais}

A Modelagem de Equações Estruturais foi estimada a partir dos Mínimos Quadrados Parciais (Partial Least Squares - PLS). Segundo Ferreira, Cabral e Saraiva (2010), a modelagem por meio dos PLS é uma técnica multivariada que difere das demais por estimar o sistema de relações lineares entre variáveis latentes a partir da combinação de construtos teóricos e medições, utilizando-se do modelo de Mínimos Quadrados Ordinários interdependentes. Inicialmente realizou-se o teste de normalidade das variáveis, em 
que se constatou que as mesmas apresentavam uma distribuição próxima do normal. Assim, com base no modelo proposto por Parker e Kohlmeyer (2005), realizou-se a análise dos dados coletados, utilizando a técnica de estimação de covariância de SEM-PLS, conforme Figura 2.

Tabela 1 Dados demográficos

\begin{tabular}{|c|c|c|c|c|c|c|c|}
\hline \multirow{9}{*}{$\begin{array}{l}\text { 윰 } \\
\text { 음 }\end{array}$} & Dados Demográficos & Masculino & $\%$ & Feminino & $\%$ & Total & $\%$ \\
\hline & 25 anos ou menos & 15 & $17,24 \%$ & 7 & $36,84 \%$ & 22 & $20,75 \%$ \\
\hline & 26 a 30 anos & 18 & $20,69 \%$ & 0 & $0,00 \%$ & 18 & $16,98 \%$ \\
\hline & 31 a 35 anos & 8 & $9,20 \%$ & 4 & $21,05 \%$ & 12 & $11,32 \%$ \\
\hline & 36 a 40 anos & 8 & $9,20 \%$ & 1 & $5,26 \%$ & 9 & $8,49 \%$ \\
\hline & 41 a 45 anos & 18 & $20,69 \%$ & 5 & $26,32 \%$ & 23 & $21,70 \%$ \\
\hline & 46 a 50 anos & 10 & $11,49 \%$ & 1 & $5,26 \%$ & 11 & $10,38 \%$ \\
\hline & 51 anos ou mais & 10 & $11,49 \%$ & 1 & $5,26 \%$ & 11 & $10,38 \%$ \\
\hline & Total & 87 & $100,00 \%$ & 19 & $100,00 \%$ & 106 & $100,00 \%$ \\
\hline \multirow{7}{*}{ 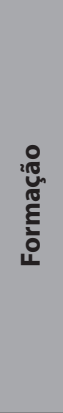 } & Ciências Contábeis & 65 & $74,71 \%$ & 14 & $73,68 \%$ & 79 & $74,53 \%$ \\
\hline & Administração & 15 & $17,24 \%$ & 2 & $10,53 \%$ & 17 & $16,04 \%$ \\
\hline & Economia & 6 & $6,90 \%$ & - & $0,00 \%$ & 6 & $5,66 \%$ \\
\hline & Engenharia & - & $0,00 \%$ & 2 & $10,53 \%$ & 2 & $1,89 \%$ \\
\hline & Sistema de Informação & 1 & $1,15 \%$ & - & $0,00 \%$ & 1 & $0,94 \%$ \\
\hline & Psicóloga & - & $0,00 \%$ & 1 & $5,26 \%$ & 1 & $0,94 \%$ \\
\hline & Total & 87 & $100,00 \%$ & 19 & $100,00 \%$ & 106 & $100,00 \%$ \\
\hline \multirow{5}{*}{$\stackrel{\bar{g}}{z}$} & Graduação & 42 & $48,28 \%$ & 15 & $78,95 \%$ & 57 & $53,77 \%$ \\
\hline & Especialização & 35 & $40,23 \%$ & 3 & $15,79 \%$ & 38 & $35,85 \%$ \\
\hline & Mestrado & 9 & $10,34 \%$ & 1 & $5,26 \%$ & 10 & $9,43 \%$ \\
\hline & Doutorado & 1 & $1,15 \%$ & - & $0,00 \%$ & 1 & $0,94 \%$ \\
\hline & Total & 87 & $100,00 \%$ & 19 & $100,00 \%$ & 106 & $100,00 \%$ \\
\hline \multirow{7}{*}{ 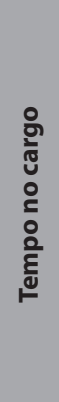 } & 5 anos ou menos & 46 & $52,87 \%$ & 13 & $68,42 \%$ & 59 & $55,66 \%$ \\
\hline & 6 a 10 anos & 20 & $22,99 \%$ & 2 & $10,53 \%$ & 22 & $20,75 \%$ \\
\hline & 11 a 15 anos & 12 & $13,79 \%$ & 1 & $5,26 \%$ & 13 & $12,26 \%$ \\
\hline & 16 a 20 anos & 8 & $9,20 \%$ & 2 & $10,53 \%$ & 10 & $9,43 \%$ \\
\hline & 21 a 25 anos & - & $0,00 \%$ & 1 & $5,26 \%$ & 1 & $0,94 \%$ \\
\hline & 25 anos ou mais & 1 & $1,15 \%$ & - & $0,00 \%$ & 1 & $0,94 \%$ \\
\hline & Total & 87 & $100,00 \%$ & 19 & $100,00 \%$ & 106 & $100,00 \%$ \\
\hline
\end{tabular}

Fonte: Dados da pesquisa. 
Figura 2 Modelo proposto com valores de $\mathrm{R}^{2}$

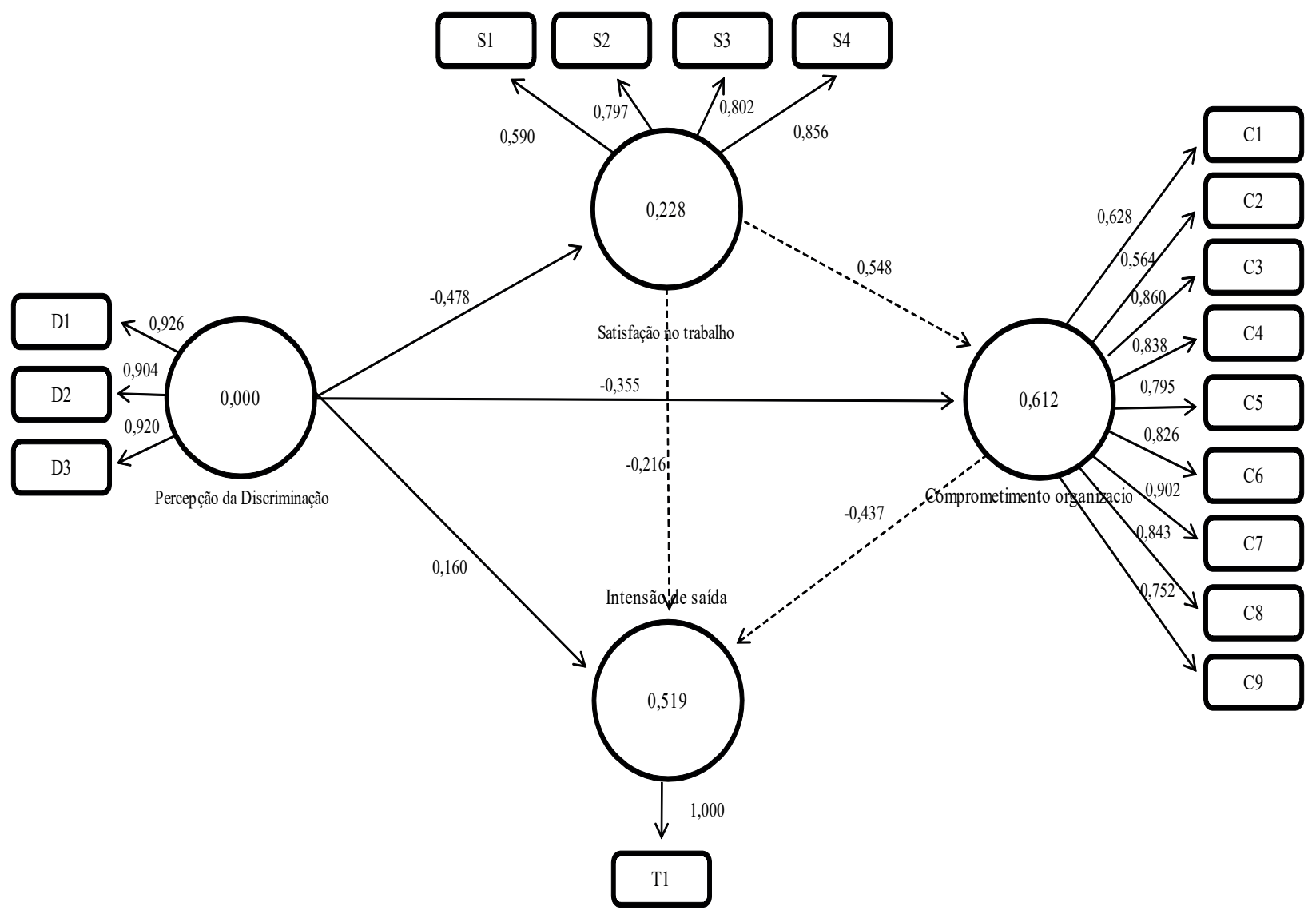

Fonte: Dados da pesquisa.

Tendo como base os critérios e procedimentos definidos pela técnica de estimação de covariância de SEM-PLS (CHIN, 1995), fez-se a adequação do modelo e realizaram-se as seguintes análises: validade convergente, validade discriminante e índice de Goodness-of-Fit.

Fornell e Larckel (1981) sugerem a utilização da Variância Média Extraída (AVE) como critério de variável latente. A AVE é utilizada para explicar a variância dos indicadores, sendo que valores acima de 0,5 indicam validade convergente suficiente (FORNELL; LARCKER, 1981; HAIR et al., 2005). Na Tabela 2, são apresentados os indicadores de estimação da validade convergente e confiabilidade do modelo.

Tabela 2 Validade convergente e confiabilidade

\begin{tabular}{l|c|c}
\multicolumn{1}{c|}{ Variáveis } & Variância Média Extraída (AVE) & Confiabilidade Composta \\
\hline Comprometimento & 0,6191 & 0,9349 \\
\hline Discriminação & 0,8403 & 0,9404 \\
\hline Satisfação & 0,5900 & 0,8498 \\
\hline Turnover & 1,0000 & 1,0000 \\
\hline
\end{tabular}

Fonte: Dados da pesquisa. 
Verifica-se, na Tabela 2, que os indicadores da AVE apresentam valores superiores a 0,5 , sugerindo a aceitação da validade do modelo. Adicionalmente, os resultados dos coeficientes de confiabilidade composta corroboram a aceitação do modelo, pois apresentam valores superiores a 0,7 , conforme recomendado por (CHIN, 1998).

$\mathrm{Na}$ análise da adequação do modelo também foi verificada a validade discriminante, que tem a finalidade de identificar até que ponto as escalas medem o que se propõem medir. Fornell e Larcker (1981) e Chin (1998) recomendam que, para estudos que utilizam a modelagem de equações estruturais, a avaliação da confiabilidade do construto validada pela confiabilidade composta apresente escores acima de 0,7. Na Tabela 3, observam-se os resultados do teste da análise discriminante.

Tabela 3 Valores de correlação entre os construtos de primeira ordem

\begin{tabular}{l|c|c|c|c}
\hline \multicolumn{1}{c|}{ Variáveis } & Comprometimento & Discriminação & Satisfação & Turnover \\
\hline Comprometimento & 1,000 & & & \\
\hline Discriminação & $-0,617$ & 1,000 & & \\
\hline Satisfação & $\mathbf{0 , 7 1 8}$ & $-0,478$ & 1,000 & \\
\hline Turnover & $-0,691$ & $\mathbf{0 , 5 3 3}$ & $-0,606$ & 1,000 \\
\hline
\end{tabular}

Fonte: Dados da pesquisa.

Os resultados apresentados na Tabela 3 demonstram não haver índices superiores a 0,85 , conforme o parâmetro sugerido por David (1998), indicando validade discriminante adequada. Para Fornell e Larcker (1981), a validade discriminante é obtida pela comparação entre a AVE e o quadrado dos coeficientes de correlação das variáveis $\left(\mathrm{R}^{2}\right)$, em que valores superiores da AVE demonstram que o modelo possui validade discriminante, conforme evidenciado na Tabela 4.

Tabela 4 Validade descriminante: Critérios Fornell e Larcker (1981)

\begin{tabular}{l|c|c|c|c} 
& Comprometimento & Discriminação & Satisfação & Turnover \\
\hline Comprometimento & $\mathbf{0 , 6 1 9}$ & & & \\
\hline Discriminação & 0,381 & $\mathbf{0 , 8 4 0}$ & & \\
\hline Satisfação & 0,516 & 0,228 & $\mathbf{0 , 5 9 0}$ & \\
\hline Turnover & 0,477 & 0,284 & 0,367 & $\mathbf{1 , 0 0 0}$ \\
\hline
\end{tabular}

Fonte: Dados da pesquisa.

Contata-se na Tabela 4 que os resultados apresentados reforçam a existência de validade discriminante do modelo. $\mathrm{O}$ valor do índice de Goodness-of-Fit (GoF) pode ser utilizado como um indicador para validar globalmente a modelagem de equações estruturais, pois considera a quantidade de variância e covariância da matriz observada. Trata-se de um índice que corresponde à média geométrica entre o $\mathrm{R}^{2}$ médio e a Variância Média Extraída (AVE).
Não há um critério mínimo para o GoF, contudo, sugere-se como adequado um GoF superior a 0,5 (TENENHAUS et al., 2005). Na Tabela 5, evidencia-se o valor do índice de Goodness-of-Fit do modelo.

Nota-se que os resultados apontados da Tabela 5 indicam um índice de GoF superior a 0,5, confirmando a validade da modelagem de equações estruturais proposta no estudo. 
Tabela 5 Avaliação do modelo estrutural pelo índice de GoF

\begin{tabular}{l|c|c}
\multicolumn{1}{c|}{ Variáveis } & $\mathbf{R}^{\mathbf{2}}$ & Variancia Média Extraida (AVE) \\
\hline Comprometimento & 0,61 & 0,6191 \\
\hline Discriminação & 0,00 & 0,8403 \\
\hline Satisfação & 0,23 & 0,5900 \\
\hline Turnover & 0,53 & 1,0000 \\
\hline GoF & R2 médio & AVE médio \\
\hline 0,53 & 0,38 & 0,7297 \\
\hline
\end{tabular}

Fonte: Dados da pesquisa.

\subsection{Análise das Relações ou Caminho (Path)}

Na Tabela 6 apresentam-se os principais resultados dos efeitos da modelagem de equações estruturais. Para avaliar a magnitude e importância dos coeficientes das relações, empregou-se a análise de bootstrap, conforme recomendado por Hair Jr et al. (2005).

Ao analisar os resultados da Tabela 6, contata-se que percepção de discriminação apresenta relação negativa e significativa ( $p$-value $<0,01)$ com satisfação do trabalho, não rejeitando a $\mathrm{H}_{1}$ (a percepção de discriminação na tomada de decisões é negativamente associada com a satisfação no trabalho). Esse resultado também foi obtido por Parker e Kohlmeyer (2005), e sugere que os auditores independentes pesquisados tendem a apresentar insatisfação no trabalho quando percebem a existência de vieses no processo de decisão da empresa de auditoria.

Tabela 6 Efeitos diretos e totais das relações entre as variáveis

\begin{tabular}{l|c|c|c|c|c|c}
\hline \multirow{2}{*}{\multicolumn{1}{c|}{ Relações entre as variáveis }} & \multicolumn{3}{c|}{ Efeitos Diretos } & \multicolumn{3}{c}{ Efeitos Totais } \\
\cline { 2 - 8 } & Valor & test t & p-value & Valor & test t & p-value \\
\hline Discriminação $\Rightarrow$ Satisfação & $-0,4775$ & 13,207 & $0,000^{* *}$ & $-0,4775$ & 13,2073 & $0,000^{* *}$ \\
\hline Discriminação $\Rightarrow$ Comprometimento & $-0,3548$ & 8,3577 & $0,000^{* *}$ & $-0,6165$ & 18,8892 & $0,000^{* *}$ \\
\hline Discriminação $\Rightarrow$ Turnover & 0,1602 & 3,4994 & $0,000^{* *}$ & 0,5330 & 13,8195 & $0,000^{* *}$ \\
\hline Satisfação $\Rightarrow$ Comprometimento & 0,5482 & 12,8440 & $0,000^{* *}$ & 0,5516 & 12,8440 & $0,000^{* *}$ \\
\hline Satisfação $\Rightarrow$ Turnover & $-0,2160$ & 6,6179 & $0,000^{* *}$ & $-0,4556$ & 12,2974 & $0,000^{* *}$ \\
\hline Comprometimento $\Rightarrow$ Turnover & $-0,4375$ & 10,6130 & $0,000^{* *}$ & $-0,4398$ & 10,6130 & $0,000^{* *}$ \\
\hline
\end{tabular}

* Significância $<0,01$

Fonte: Dados da pesquisa.

A percepção de discriminação apresenta relação negativa e significativa $(p$-value $<0,01)$ com o comprometimento organizacional e relação positiva e significativa com a intenção de o indivíduo deixar a empresa (turnover). Isso indica que as decisões tendenciosas percebidas pelos auditores reduzem seu comprometimento com a organização e influenciam a sua saída da empresa. Esses resultados não rejeitam as hipóteses $\mathrm{H}_{2}$ (a percepção de discriminação na tomada de decisões é negativamente associada com o comprometimento organizacional) e $\mathrm{H}_{3}$ (a percepção de discriminação na tomada de decisões é positivamente associada com a intenção de o indivíduo deixar a empresa).

Verifica-se, também, relação positiva e significativa $(p$-value $<0,01)$ entre a satisfação no trabalho 
e o comprometimento organizacional. Isso sugere que os auditores satisfeitos com seu emprego tendem a apresentar maior comprometimento com a organização. Por outro lado, os resultados indicam que a satisfação influencia negativamente a intenção dos auditores em saírem da empresa. Decorre que os auditores, quando satisfeitos com o trabalho, tendem a permanecer no emprego.

A mesma lógica é aplicada para a relação negativa encontrada entre o comprometimento organizacional e o turnover. Essa relação indica que quando os profissionais de auditoria estão comprometidos com a organização, sua saída da empresa é pouco frequente. Esses achados convergem com os resultados Geremia, Kanan e Marcon (2018), que indicam que trabalhar em uma organização justa pode significar maior possibilidade de permanência diante de outra oportunidade de emprego.

\section{CONCLUSÕES}

O estudo objetivou verificar a relação da percepção de justiça organizacional com satisfação no trabalho, comprometimento organizacional e intenção de turnover de auditores de empresas de auditoria. Os resultados da pesquisa demonstram que a percepção de justiça organizacional influencia significativamente a satisfação no trabalho, o comprometimento organizacional e a intenção de saída do auditor da organização.

Conforme apresentado pela literatura, a justiça organizacional é um fator positivo no comportamento do indivíduo dentro da organização. Quando este percebe indícios de injustiça, há efeitos negativos em sua relação com a organização. A percepção de justiça organizacional tende a contribuir para a eficiência da empresa, evitando que funcionários qualificados e motivados saiam para trabalhar em organizações concorrentes.

Em estudos anteriores, como o de Paker e Kohlmeyer (2005), foi constatado que as situações de injustiça organizacional se refletem negativamente no comprometimento com a organização e na satisfação no trabalho. No presente estudo observou-se também a existência de uma forte relação entre a percepção de justiça e a intenção de saída dos auditores das empresas em que trabalham. Esses resultados auxiliam na compreensão de aspectos pelos quais decisões tendenciosas geram maior rotatividade nas empresas de auditoria. Conforme Geremia, Kanan e Marcon (2018), é possível considerar que a percepção de justiça é importante para a permanência do funcionário na empresa, pois, se ele não percebe justiça organizacional, apresenta maior probabilidade de deixar a empresa na qual trabalha.

Portanto, conclui-se que a justiça é um importante elemento organizacional que, quando exercida, tende a produzir resultados positivos para a organização. No caso específico do campo investigado, pode proporcionar um ambiente saudável para que auditores consigam exercer suas funções com satisfação no trabalho, comprometimento organizacional e com baixa intenção de turnover. Por outro lado, decisões tendenciosas dos gestores das empresas de auditoria podem reduzir a satisfação e o comprometimento dos auditores com o trabalho, além de aumentar as chances de o profissional deixar a organização.

Ressalta-se, no entanto, que os resultados obtidos se limitam à amostra pesquisada, ao momento de resposta do questionário, que pode variar com a circunstância, e à metodologia utilizada. Assim, recomenda-se, para estudos futuros, a pesquisa de outros campos, envolvendo profissionais com exigências de qualificação visíveis ao público, como docentes de instituições de ensino superior. Outras variáveis podem ser relacionadas com justiça organizacional, ou ainda, caminhos diferentes podem ser identificados na modelagem de equações estruturais.

\section{REFERÊNCIAS}

ADAMS, J. S. Inequity in social exchange. In: BERKOWITZ, L. (Ed.). Advances in experimental social psychology. 2 ed. New York: Academic Press, 1965. pp 267-299.

ALEXANDER, S.; RUDERMAN, M. The role of procedural and distributive justice in organizational behavior. Social Justice Research, v.1. n. 2, p. 177198, 1987. 
ALI, N.; JAN, S. Relationship between organizational justice and organizational commitment and turnover internations amongst medical representative of pharmaceuticals companies of Pakistan. Journal of Managerial Sciences, v. 6, n. 2, p. 201-212, 2012.

ALMEIDA, G. O.; MONTEIRO DA SILVA, A. M. M. Justiça organizacional, impactos no burnout e comprometimento dos trabalhadores. GESTÃO.Org., v. 4, p. $160-175,2006$.

ASSMAR, E. M. L.; FERREIRA, M. C.; SOUTO, S. O. Justiça organizacional: Uma revisão crítica da literatura. Psicologia: Reflexão e Crítica, v. 18, n. 3, p. 443-453, 2005.

BENTLER, P. M.; CHOU, C. P. Practical issues in structural modeling. Sociological Methods Research, v. 16, n. 78, p. 78-117, 1987.

BEUREN, I. M.; ALTOÉ, S, M. L.; DAL VESCO, D. G. Percepção de justiça e comportamento de cidadania organizacional: Um estudo na perspectiva do sistema de mensuração de desempenho estratégico. Cuadernos Administración, v. 28, n. 51, p. 133-157, 2015.

BEUREN, I. M.; KLEIN, L.; LARA, F. L.; ALMEIDA, L. B.; Percepção de justiça nos sistemas de controle gerencial aumenta comprometimento e confiança dos gestores? Revista de Administração Contemporânea, v. 20, n. 2, p. 216-237, 2016.

BEUREN, I. M.; SANTOS, V. Percepção da justiça organizacional na avaliação de desempenho de controllers. Enfoque Reflexão Contábil, v. 31, n. 3, p. 53-72, 2012.

BIES, R. J.; MOAG, J. F. Interactional justice: Communication criteria of fairness. In: LEWICKI, R. J.; SHEPPARD, B. H.; BAZERMAN, M. (Eds.). Research on negotiation in organizations. Greenwich, CT: JAI Press, 1986. pp 43-55.
BORGES, M. S.; RAMOS, N. M. Turnover: Uma consequência de estratégias ineficientes de gestão empresarial? In: CONGRESSO VIRTUAL BRASILEIRO DE ADMINISTRAÇÃO, 8., 2011. Anais ... Convibra, 2011.

BREI, V. A.; LIBERALI NETO, G. O uso da técnica de modelagem em equações estruturais na área de marketing: Um estudo comparativo entre publicações no Brasil e exterior. Revista de Administração Contemporânea, v. 10, n. 4, p. 131-151, 2006.

CAMPOS, C. V. A.; MALIK, A. M. Satisfação no trabalho e rotatividade dos médicos do Programa de Saúde da Família. Revista de Administração Pública, v. 42. n. 2. p. 347-368, 2008.

CHIN, W. W. Partial least squares is to LISREL as principal components analysis is to common factor analysis. Technology Studies, v. 2, n. 1, p. 315-319, 1995.

CHIN, W. W. The Partial Least Squares approach to Structural Equation Modeling. In: MARCOULIDES, G. A. (Ed.). Modern methods for business research. USA: Lawrence Erlbaum Associates, 1998. pp 295-336.

CHOI, S. Organizational justice and employee work attitudes: The federal case. The American Review of Public Administration, v. 41, n. 2, p. 185-204, 2011.

COHEN-CHARASH, Y.; SPECTOR, P. E. The role of justice in organizations: A meta analysis. Organizational Behavior and Human Decision Processes, v. 86, n. 2, p. 278-321, 2001.

COLQUITT, J. A.; CONLON, D. E.; WESSON, M. J.; PORTER, C. O. L. H.; NG, K. Y. Justice at the millennium: A meta-analytic review of 25 years of organizational justice research. Journal of Applied Psychology, v. 86, n. 3, p. 425-445, 2001.

CROPANZANO, R.; BOWEN, D. E.; GILLILAND, S. $\mathrm{W}$. The management of organization justice. Academy of Management Perspectives, v. 21, n. 4, p. 34-48, 2007. 
CROPANZANO, R.; GREENBERG, J. Progress in organizational justice: Tunneling through the maze. In: COOPER, C. L.; ROBERTSON, I. T. (Eds.). International Review of Industrial and Organizational Psychology. New York: John Wiley \& Sons, 1997. pp 317-372.

FERREIRA, I.; CABRAL, J.; SARAIVA, P. An integrated framework based on the ECSI approach to link mould customers' satisfaction and product design. Total Quality Management \& Business Excellence, v. 21, n. 12 , p. $1383-1401,2010$.

FOLGER, R.; CROPANZANO, R. Organizational justice and human resource management. Thousand Oaks, CA: Sage, 1998.

FOLGER, R.; KONOVSKY, M. A. Effects of procedural and distributive justice on reactions to pay raise decisions. Academy of Management Journal, v. 32, n. 1, p. 115-130, 1989.

FORNELL, C.; LARCKER, D. Structural equation models with unobservable variables and measurement error: Algebra and statistics. Journal of Marketing Research, v. 18, n. 3, p. 328-388, 1981.

GEREMIA, H. C.; KANAN, L. A.; MARCON, S. R. A. Organizational justice, turnover \& information technology professional. Psicologia desde el Caribe, v. 35, p. 224-241, 2018.

GIRAUD, F.; LANGEVIN, P.; MENDOZA, C. Justice as a rationale for the controllability principle: A study of managers' opinions. Management Accounting Research, v. 19, n. 1, p. 32-44, 2008.

GREENBERG, J. The intellectual adolescence of organizational justice: You've come a long way, maybe. Social Justice Research, v. 6, n. 1, p. 135-148, 1993.

HAIR JR., J. F.; ANDERSON, R. E.; TATHAM, R. L.; BLACK, W. C. Análise multivariada de dados. 5. ed. Porto Alegre: Bookman, 2005.
HAIR JR, J. F.; HULT, G. T. M.; RINGLE, C.; SARSTEDT, M. A primer on partial least squares structural equation modeling. Los Angeles: Sage, 2014.

HOMANS, G. Social Behaviour: Its elementary forms. London: Routledge \& Kegan Paul, 1961.

KONOVSKY, M. A.; CROPANZANO, R. Perceived fairness of employee drug testing as a predictor of employee attitudes and job performance. Journal of Applied Psychology, v. 76, n. 5, p. 698-707, 1991.

HOPKINS, S. M; WEATHINGTON, B. L.The relationships between justice perceptions, trust, and employee attitudes in a downsized organization. The Journal of Psychology, v. 140, n. 5, p. 477-498, 2006.

HOPPOCK, R. Job satisfaction. New York: Harper and Row, 1935.

HUNTON, J.; NEIDERMEYER, P.; WEIR, B. Hierarchical and gender differences in private accounting practice. Accounting Horizons, v. 10, n. 2, p. 14-31, 1996.

JESUS, R. G.; ROWE, D. E. O. Percepção de políticas de gestão de pessoas e comprometimento organizacional: O papel mediador da percepção de justiça organizacional. Tourism \& Management Studies, v. 11, n. 2, p. 211-218, 2015.

LEOHLIN, J. C. Latent variable models: An introduction to factor, path, and structural equation analysis. 4 ed. New Jersey: Mahwah, 2004.

LEVENTHAL, G. S. What should be done with equity theory? New approaches to the study of fairness in social relationships. In: GERGEN, K.; GREENBERG, M.; WILLIS, R. (Eds.) Social exchange: New advances in theory and research. New York: Plenum Press, 1980. pp 27-55. 
LOCKE, E. The nature and causes of job satisfaction. In: DUNNETTE, M. D. (Ed.). Handbook of industrial and organizational psychology. Universidade de Michigan: Consulting Psychologists Press, 1976. pp 1297-1350.

LONDON, M.; HOWAT, G. The relationship between commitment and conflict resolution behavior. Journal of Vocational Behavior, v. 13, n. 1, p. 1-14, 1978.

MAIA, A. F. O gestor e a justiça organizacional. 2005. 82f. Dissertação (Mestrado em Administração de Empresas) - Fundação Getulio Vargas, São Paulo, 2005.

MCNICHOLS, C.; STAHL, M.; MANLEY, T. A validation of Hoppock's job satisfaction measure. Academy of Management Journal, v. 21, n. 4, p. 737-742, 1978.

MILLER, C. L.; SIEGEL, P. H.; REINSTEIN, A. Auditor and no-mentor supervisor relationships: Effects of mentoring and organizational justice. Managerial Auditing Journal, v. 26, n. 1, p. 5-31, 2011.

MIKULA, G. The experience of injustice: Toward a better understanding of its phenomenology. In: BIERHOFF, H. W.; COHEN, R. L.; GREENBERG, J. (Eds.). Justice in social relations. New York: Plenum, 1986. pp 103-124.

MOWDAY, R.; STEERS, R.; PORTER, L. The measurement of organizational commitment. Journal of Vocational Behavior, v. 14, n. 2, p. 224-247, 1979.

MUZUMDAR, P. Influence of interactional justice on the turnover behavioral decision in an organization. Journal of Behavioral Studies in Business, v. 4, n. 4, p. 1-11. 2011.

OMAR, A. Justice organizacional, individualismo-colectivismo y estrês laboral. Psicologia y Salud, v. 16, n. 2, p. 207-217, 2006.
PARKER, R. J.; KOHLMEYER, J. M. Organizational justice and turnover in public accounting firms: A research note. Accounting, Organizations and Society, v. 30, n. 4, p. 357-369, 2005.

PONNU, C. H.; CHUAH, C. C. Organizational commitment, organizational justice and employee turnover in Malaysia. African Journal of Bussiness Management, v. 4, n. 13, p. 2676-2692, 2010.

REGO, A.; SOUTO, S. A percepção de justiça como antecedentes do comprometimento organizacional: Um estudo luso-brasileiro. Revista de Administração Contemporânea, v. 8, n. 1, p. 151-177, 2004.

RINGLE, C. M.; SILVA, D.; BIDO, D. Modelagem de equações estruturais com a utilização do SmartPLS. Revista Brasileira de Marketing, v. 13, n. 2, p. 5673, 2014.

ROCHA, M. S.; PAIVA, K. C. M. Justiça organizacional, atitudes retaliatórias e o comprometimento organizacional: Um estudo comparativo longitudinal com Jovens Trabalhadores. In: CONGRESSO BRASILEIRO DE ESTUDOS ORGANIZACIONAIS, 4., 2016. Anais ... CBEO, 2016.

SKARLICKI, D. P.; FOLGER, R. Retaliation in the Workplace: The roles of distributive procedural, and interactional justice. Journal of applied Psychology, v. 82, n. 3, p. 434- 443, 1997.

SIEGEL, P. H.; REINSTEIN, A.; MILLER, C. L. Mentoring and organizational justice among audit professionals. Journal Accounting Auditing \& Finance, v. 16, n. 1, p. 1-25, 2001.

SOTOMAYOR, A. M. S. B. Avaliação de desempenho e compromisso organizacional: A perspectiva da justiça organizacional. Revista Universo Contábil, v. 3, n. 3, p. 87-100, 2007.

STEVENS, J. Applied multivariate statistics for the social science. 4. ed. New Jersey: Mahwah, 2002. 
TENENHAUS, M.; VINZI, V.; CHATELIN, Y.; LAURO, C. PLS path modeling. Computacional Statistics \& Data Analysis, v. 48, n. 1, p. 159-205, 2005.

THIBAUT, J.; WALKER, L. Procedural justice: A psychological analysis. Hillsdale, NJ: Erlbaum, 1975.

TYLER, T.; LIND, E. A relational model of authority in groups. Advances in Experimental Social Psychology, v. 25, n. 1, p. 115-191, 1992. 


\section{APÊNDICE A INSTRUMENTO DE PESQUISA}

\begin{tabular}{|c|c|}
\hline Questões do instrumento de pesquisa & Autores \\
\hline $\begin{array}{l}\text { Intenção de turnover } \\
\text { 1) Salvo qualquer imprevisto tenho a intenção de ficar na minha empresa atual. }\end{array}$ & $\begin{array}{l}\text { London e } \\
\text { Howat (1978) }\end{array}$ \\
\hline $\begin{array}{l}\text { Percepção de discriminação } \\
\text { 1) Eu acredito que meu atual empregador me discrimina em relação a promoções e oportunidades. } \\
\text { 2) Eu acredito que o meu atual empregador me discrimina a respeito das atribuições de tarefas e de trabalho. } \\
\text { 3) Eu acredito que o meu atual empregador me discrimina em relação a atual recompensa (remuneração). }\end{array}$ & $\begin{array}{l}\text { Hunton, } \\
\text { Neidermeyer e } \\
\text { Weir (1996) }\end{array}$ \\
\hline $\begin{array}{l}\text { Comprometimento organizacional } \\
\text { 1) Eu falo dessa organização para meus amigos como uma grande organização para se trabalhar. } \\
\text { 2) Eu aceitaria quase qualquer tipo de atribuição de trabalho, a fim de continuar trabalhando para esta organização. } \\
\text { 3) Eu estou extremamente feliz por ter escolhido esta organização para trabalhar ao invés de outras que eu estava } \\
\text { pensando no momento em que eu ingressei. } \\
\text { 4) Para mim, esta é a melhor de todas as organizações possíveis para a qual trabalhei. } \\
\text { 5) Eu realmente me importo sobre o destino desta organização. } \\
\text { 6) Eu acho que os meus valores e os valores da organização são muito semelhantes. } \\
\text { 7) Tenho orgulho de dizer aos outros que faço parte desta empresa. } \\
\text { 8) Esta organização realmente inspira o melhor em mim na forma de desempenho no trabalho. } \\
\text { 9) Estou disposto a me esforçar além do que é normalmente esperado, a fim de ajudar esta organização a ser bem } \\
\text { sucedida. }\end{array}$ & $\begin{array}{l}\text { Mowday, Steers e } \\
\text { Porter (1979) }\end{array}$ \\
\hline $\begin{array}{l}\text { Satisfação no Trabalho } \\
\text { 1) Qual das afirmações seguintes, melhor expressa como você se sente em relação à mudança de seu trabalho? } \\
\text { (1) Eu gostaria de terminar meu trabalho de uma só vez, se pudesse. } \\
\text { (2) Eu gostaria de ter o trabalho de outra pessoa, desde que eu pudesse ganhar tanto quanto eu estou ganhando } \\
\text { agora. } \\
\text { (3) Eu gostaria de mudar meu trabalho. } \\
\text { (4) Eu trocaria meu emprego atual por outro. } \\
\text { (5) Eu não estou interessado em mudar de trabalho, mas eu faria isso se pudesse conseguir um emprego melhor. } \\
\text { (6) Eu não consigo pensar em nenhum trabalho pelo qual eu trocaria o atual. } \\
\text { (7) Eu não trocaria o meu trabalho atual por outro. }\end{array}$ & Hoppock (1935) \\
\hline $\begin{array}{l}\text { 2) Escolha uma das seguintes afirmações que melhor representa quanto você gosta do seu trabalho. } \\
\text { (1) Eu odeio ele. } \\
\text { (2) Eu não suporto ele. } \\
\text { (3) Eu não gosto dele. } \\
\text { (4) Eu sou indiferente a ele. } \\
\text { (5) Eu gosto dele. } \\
\text { (6) Estou entusiasmado com ele. } \\
\text { (7) Eu amo ele. }\end{array}$ & \\
\hline $\begin{array}{l}\text { 3) Qual das seguintes afirmações mostra como você se considera comparativamente com outras pessoas? } \\
\text { (1) Ninguém não gosta de seu trabalho mais do que eu não gosto do meu. } \\
\text { (2) Eu não gosto do meu trabalho muito mais do que a maioria das pessoas não gosta do trabalho delas. } \\
\text { (3) Eu não gosto do meu trabalho mais do que a maioria das pessoas não gosta do trabalho delas. (4) Eu gosto do } \\
\text { meu trabalho tanto quanto a maioria das pessoas gosta do delas. } \\
\text { (4) Eu gosto do meu trabalho mais do que a maioria das pessoas gosta do delas. } \\
\text { (5) Eu gosto do meu trabalho muito mais do que a maioria das pessoas gosta do delas. (7) Ninguém gosta de seu } \\
\text { trabalho mais do que eu gosto do meu. }\end{array}$ & \\
\hline $\begin{array}{l}\text { 4) Qual das seguintes afirmações mostra como a maior parte do tempo você se sente satisfeito com o seu trabalho? } \\
\text { (1) Nunca. } \\
\text { (2) Raramente. } \\
\text { (3) Ocasionalmente. } \\
\text { (4) Cerca de metade do tempo. } \\
\text { (5) Uma boa parte do tempo. } \\
\text { (6) A maior parte do tempo. } \\
\text { (7) O tempo todo. }\end{array}$ & \\
\hline
\end{tabular}

Fonte: Adaptado de Parker e Kohlmeyer (2005). 